\title{
FATORES QUE AFETAM A FIXAÇÃO DO FOSFORO - I - PERÍODO DE INCUBAÇÃO*
}

\author{
Francisco José de Albuquerque Cavalcanti** \\ Francisco de Assis Ferraz de Mello***
}

\begin{abstract}
Amostras da camada arável de 5 séries de solos do município de Piracicaba, S. Paulo, foram tratadas com solução de fosfato monocálcico $(4 \mathrm{ml}$ de solução contendo $1.500 \mathrm{ppm}$ de $P$ para $10 \mathrm{~g}$ de terra), seguindo-se incubação por diferentes períados $\left(T_{0}=0 ; T_{1}=2 ; T_{3}=200\right.$ horas $)$. Dos solos utilizados, 3 eram Latossolos e 2 Podzólicos. Ao término de cada perído de incubação determinou-se o teor de $\mathrm{P}$ extraido com solução $0,025 \mathrm{~N}$ em $\mathrm{H}_{2} \mathrm{SO}_{4}$ e $0,05 \mathrm{~N}$ em $\mathrm{HCl}$.

A quantidade de $\mathrm{P}$ fixada foi obtida subtraindo-se do "extrato corrigido" para $T_{0}$ as concentrações encontradas para $T_{1}, T_{2}$ e $T_{3}$.

Para se proceder tal correção foi necessário tratar cadá amostra de terra com uma quantidade de água destilada igual à da solução empregada.

A análise estatística indicou, para cada solo, uma significância ao nível de $1 \%$ para os cfeitos linear e quadrático do período de incubação.

A análise conjunta para as 5 amostras revelou a ausência de diferenças significativas entre os Latossolos e de diferenças significativas entre os Podzólicos.
\end{abstract}

\section{INTRODUÇAO}

Quando se procura estudar a fixação do $P$ é costume seperar os processos que predominam em solos ácidos dos que predominam em solos alcalinos, mesmo sabendo-se que todos eles podem atuar sob quaisquer condições de $\mathrm{pH}$.

* Trabalho realizado com parte dos dados da Dissertação apresentada pelo primeiro autor à E.S.A. "Luiz de Queiroz", USP, em 1974. Entregue para publicação em: 27-12-1977.

* Eng.` Agr.०, Mestre em Solos e Nutrição de Plantas, da Seção de Solos do Instituto do Pesquisas Agropecuária do Nordeste (IPEANE), Recife, Pernambuco.

*** Prof. Adjunto do Departamento de Solos, Geologia e Fertilizantes, ESALQ - USP. 
$\mathrm{O}$ pH do solo chega a ser um fator tão importante na fixação do $\mathrm{P}$ que MALAVOLTA et al. (1965) lembram a possibilidade de sua elevação acarretar um aumento no teor de fósforo disponível de tal ordem que possa desaparecer, por um certo tempo, a necessidade de se aplicar fertilizantes fosfatados.

Além desse fator, vários outros afetam a retenção do $\mathrm{P}$ pelo solo: A solubilidade da forma em que esse elemento é adicionado também poderá contribuir para uma fixação mais ou menos acentuada. Em solos com elevada capacidade de fixação Mc LEAN \& LOGAN (1970) mostraram que a concentração de $\mathrm{P}$ em plântulas de milho decresceu com o aumento da solubilidade em água do fertilizante utilizado.

$O$ excesso de umidade parece reduzir a retenção do $\mathbf{P}$. Em condições de alagamento, os fosfatos féricos são reduzidos para compostos ferrosos, mais solúveis (GOEDERT et al., 1971 e MANDAL \& DIAS, 1970) .

O efeito do tempo de contacto do solo com o íon fosfato, objetivo deste trabalho, foi estudado de modo tão detalhado por BASE \& SHIELING (1950) que chegaram a estabelecer períodos diferindo apenas de uma hora.

$\mathrm{A}$ redução da quantidade de $\mathrm{P}$ disponível às plantas, devido à incubação do solo ocorre principalmente em regiões de clima quente (ENGELSTAD \& ALLEN, 1971).

Neste ensaio, efetuado com amostras representativas da camada arável de 5 séries de solos da região de Piracicaba, São Paulo, procurou-se estimar o efeito do período de incubação sobre a porcentagem de $\mathrm{P}$ fixado.

\section{MATERIAL E MÉTODOS}

As amostras, numeradas de I a V (Quadro 1), foram coletadas na região de iPracicaba, São Paulo, tendo a parte experimental sido realizada nos laboratórios do Departamento de Solos, Geologia e Fertilizantes, da Escola Superior de Agricultura "Luiz de Queiroz", da Universidade de São Paulo.

QUADRO 1 - Classificação dos solos utilizados (adaptado de RANZANI et al, 1966).

\begin{tabular}{rlll}
\hline & & \multicolumn{2}{c}{ Grande Grupo } \\
\cline { 3 - 4 } Amostra & \multicolumn{1}{c}{ Série } & $\begin{array}{c}\text { (Comissão de } \\
\text { Solos-CNEPA) }\end{array}$ & (7. Aproximação) \\
\hline I & Guamium & Latossolo & Haplacrox \\
II & Iracema & Latossolo & Haplacrox \\
III & Gibóia & Podzólico & Typustalf \\
IV & Ibitiruna & Podzólico & Typochrult \\
V & Luiz de Queiroz & Latossolo & Ultustalf \\
\hline
\end{tabular}


A amostra denominada Solo I foi tomada perto do perfil $\mathrm{P}_{2}$, estudado por ANDRADE (1971). As demais, Solos II a V, foram coletadas, respectivamente, nas proximidades dos perfis $\mathrm{P}_{20}, \mathrm{P}_{26}, \mathrm{P}_{28}$ e $\mathrm{P}_{33}$, descritos por RANZANI et al (1966).

Após serem secas ao ar, passadas em peneira de $2 \mathrm{~mm}$ e analisadas (Quadros 2 e 3), tomou-se, para cada tratamento, o equivalente a $10 \mathrm{~g}$ de TFSE ao qual se adicionou $4 \mathrm{ml}$ de uma solução de fosfato monocálcico contendo $1.500 \mathrm{ppm}$ de P (WAUGH \& FITTS, 1966). Para tratamentos, repetidos 4 vezes, foram estabelecidos os seguintes períodos de incubação: $\mathrm{T}_{0}=0 ; \mathrm{T}_{1}=2 ; \mathrm{T}_{3}=200$ horas.

QUADRO 2 - Análise mecânica dos solos utilizados

\begin{tabular}{|c|c|c|c|c|}
\hline \multirow[b]{2}{*}{ Amostra } & \multicolumn{3}{|c|}{ Porcentagem } & \multirow[b]{2}{*}{ Classe Textural } \\
\hline & $\begin{array}{c}\text { Areia } \\
(2-0,05 \\
\mathrm{mm})\end{array}$ & $\begin{array}{c}\text { Limo } \\
(0,05-0,002 \\
\mathrm{mm})\end{array}$ & $\begin{array}{c}\text { Argila } \\
<0,002 \mathrm{~mm}\end{array}$ & \\
\hline I & 19,8 & $17,3:$ & 62,9 & Argiloso \\
\hline II & 23,7 & 30,4 & 45,9 & $\begin{array}{l}\text { Argiloso } \\
\text { Arenoso }\end{array}$ \\
\hline III & 73,3 & 20,2 . & 6,5 & franco \\
\hline IV & 88,0 & 5,8 & 6,2 & Arenoso \\
\hline 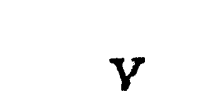 & 35,3 & 26,0 & 38,7 & $\begin{array}{l}\text { Franco } \\
\text { argiloso }\end{array}$ \\
\hline
\end{tabular}

A reposição da água perdida no decorrer do ensaio foi feita ccm base no peso inicial de cada recipiente. Findo o tempo de incubação previsto para cada tratamento, procedeu-se à determinação do teor de $\mathbf{P}$ do extrato obtido com uma solução $0,025 \mathrm{~N}$ em $\mathrm{H}_{2} \mathrm{SO}_{4}$ e $0,05 \mathrm{~N}$ em $\mathrm{HCl}$ (VETTORI, 1966) .

A quantidade de $\mathrm{P}$ fixada foi obtida subtraindo-se do "extrato corrigido" para $\mathrm{T}_{0}$ as concentrações corrigidas para $\mathrm{T}_{1}, \mathrm{~T}_{2} \mathrm{e} \mathrm{T}_{3}$. Os resultados assim conseguidos foram, a seguir, transformados em $\mathrm{mg}$ de $\mathbf{P}$ por $100 \mathrm{~g}$ de terra.

Para se proceder à correção dos valores inicialmente encontrados foi preciso tratar cada amostra de solo com uma quantidade de água destilada idêntica à da solução empregada e por período iguais aos observados (2, 4 e 200 horas).

Os resultados assim obtidos foram submetidos à análise estatística, com o objetivo de pesquisar, para cada solo, os efeitos linear e quadrático dos períodos de incubação estabelecidos. A seguir, foi feita uma análise conjunta, visando a classificação dos solos segundo as quantidades de $\mathrm{P}$ fixadas. 


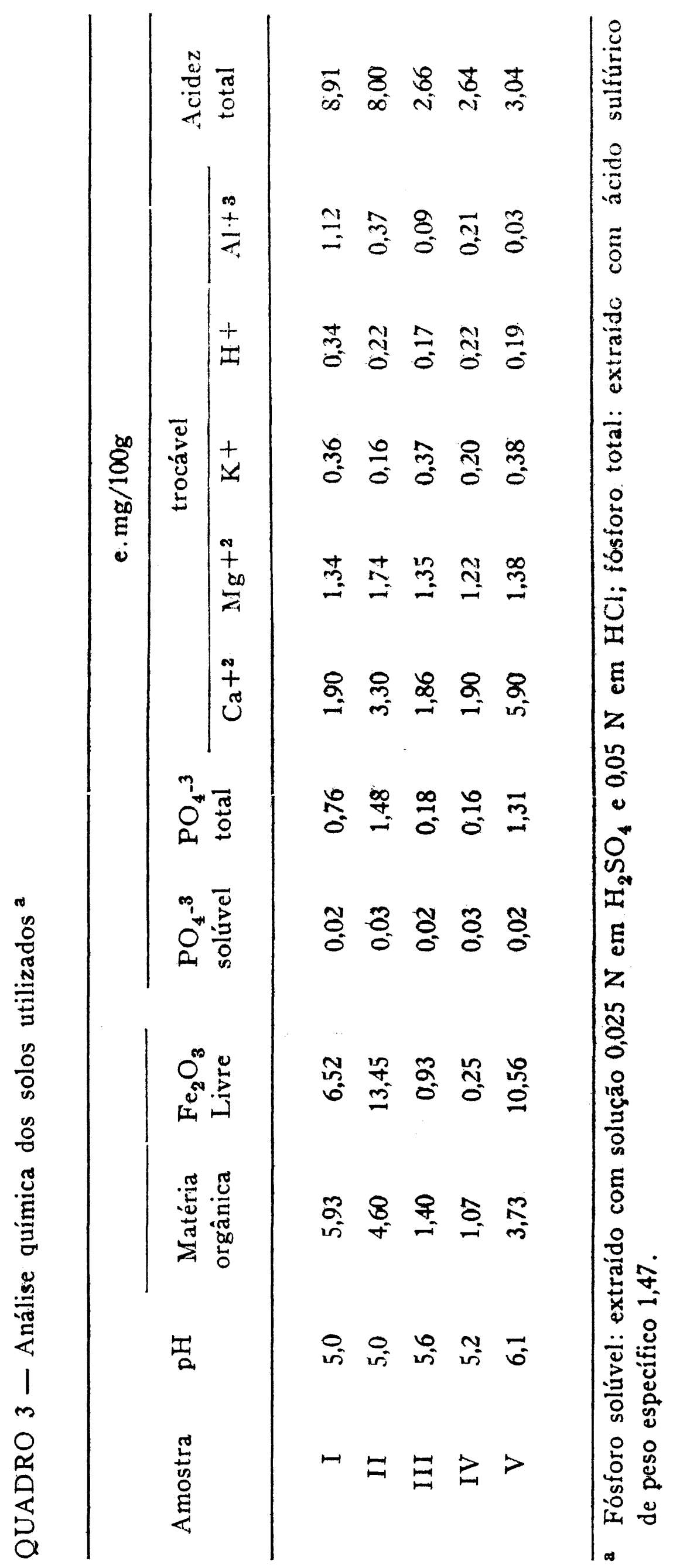




\section{RESULTADOS E DISCUSSÃO}

As concentrações dos extratos obtidos, tanto do material tratado com solução de fosfato monocálcico como das amostras que receberam $4 \mathrm{ml}$ de água destilada (Quadros 4 e 5 , respectivamente), permitiram a elaboração do Quadro 6, relativo ao extrato corrigido.

As quantidades de $\mathrm{P}$ fixadas por cada solo, nos períodos de $2,20 \mathrm{e}$ 200 horas podem ser vistas no Quadro 7. Para uma comparação mais fácil entre os resultados proporcionados pelos diferentes solos empregados pode-se recorrer às curvas da Fig. 1 .

A partir dos resultados da análise estatística para cada solo de per sí (Quadro 8) ficou constatado, ao desdobrarem-se os graus de liberdade, que os efeitos linear e quadrático dos períodos de incubação foram altamente significativos, independente da natureza da amostra.

A análise conjunta para as 5 amostras revelou diferença significativa para tratamentos $\left(F=36,92^{* *}\right)$ e o teste de Tukey a $5 \%$ mostrou a ausência de diferenças significativas entre os solos I, II e V (Latossolos), o mesmo não acontecendo entre os de número III e IV (Podzólicos), como pode ser observado no Quadro 9.

Esses resultados acham-se intimamente relacionados com algumas das características apresentadas pelos solos em questão. Assisim, levando-se em conta a análise mecânica (Quadro 2), observa-se um teor de argila de 6 a 10 vezes mais baixo nos solos III e IVे em relação aos solos I, II e V.

Dos resultados da análise química (Quadro III), aqueles referentes aos teores de $\mathrm{Fe}_{2} \mathrm{O}_{3}$ livre prestam-se bem para comparações: No caso, ao mais alto teor de $\mathrm{Fe}_{2} \mathrm{O}_{3}$ livre (Solo II - 13,45\%) corresponde a uma maior quantidade de $\mathrm{P}$ fixado, enquanto que a mais baixa porcentagem de óxido de ferro livre (Solo IV - 0,25\%) corresponde exatamente à amostra que, pelo teste de Tukey a $5 \%$, fixou a menor quantidade de fósforo.

\section{CONCLUSÕES}

Com base na análise estatística dos dados obtidos para as condições em que foi realizado o presente trabalho, são válidas as seguintes conclusões:

a) dentro de cada solo, a quantidade de $\mathrm{P}$ fixada aumentou com o tempo de incubação;

b) houve efeitos linear e quadrático do tempo de incubação sobre a quantidade de $P$ fixado;

c) os Latossolos não diferiram entre sí em relação às quantidades de $\mathrm{P}$ fixadas, ocorrendo o inverso entre os Podzólicos;

d) os Latossolos fixaram mais P que os Podzólicos. 


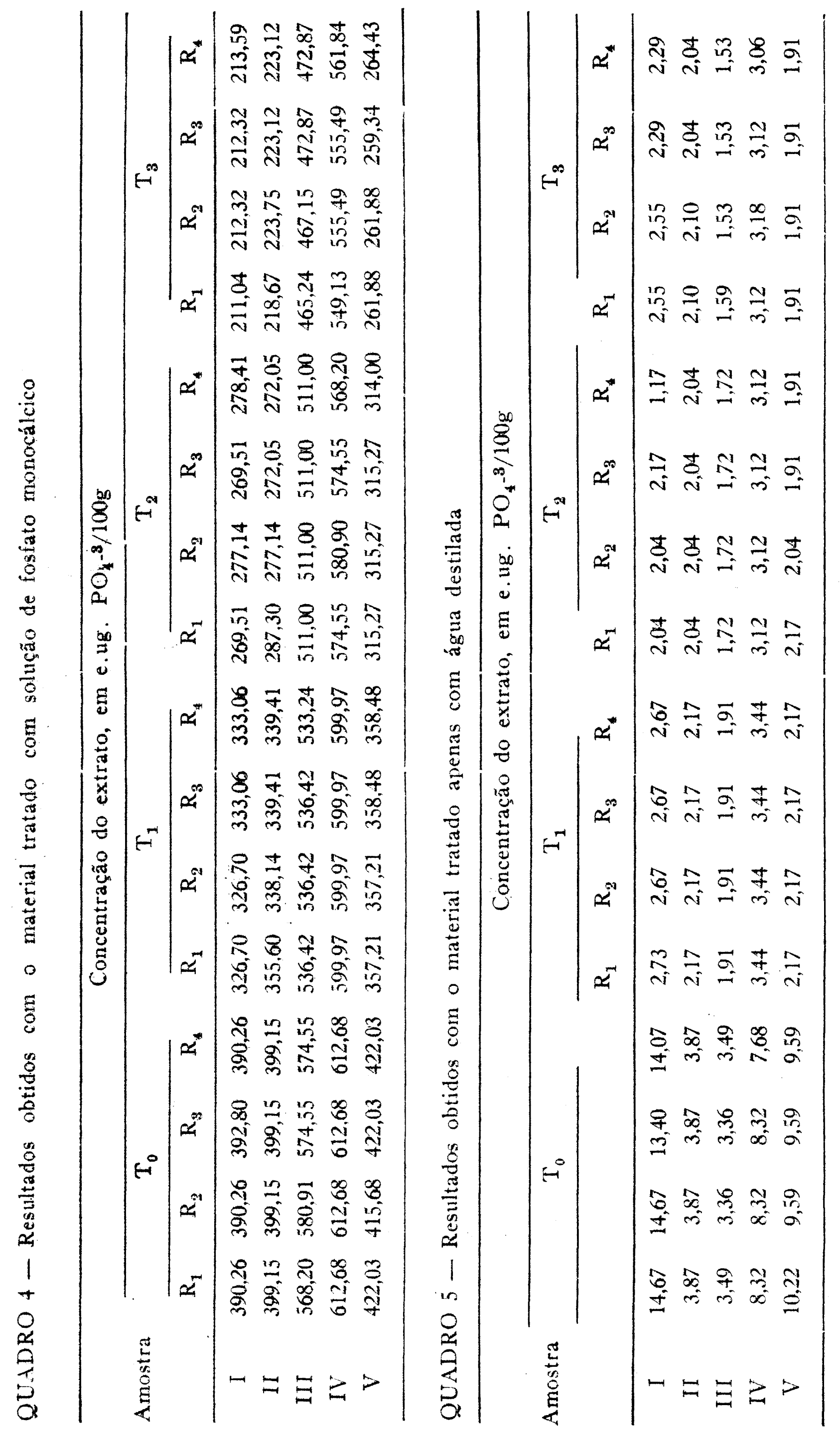




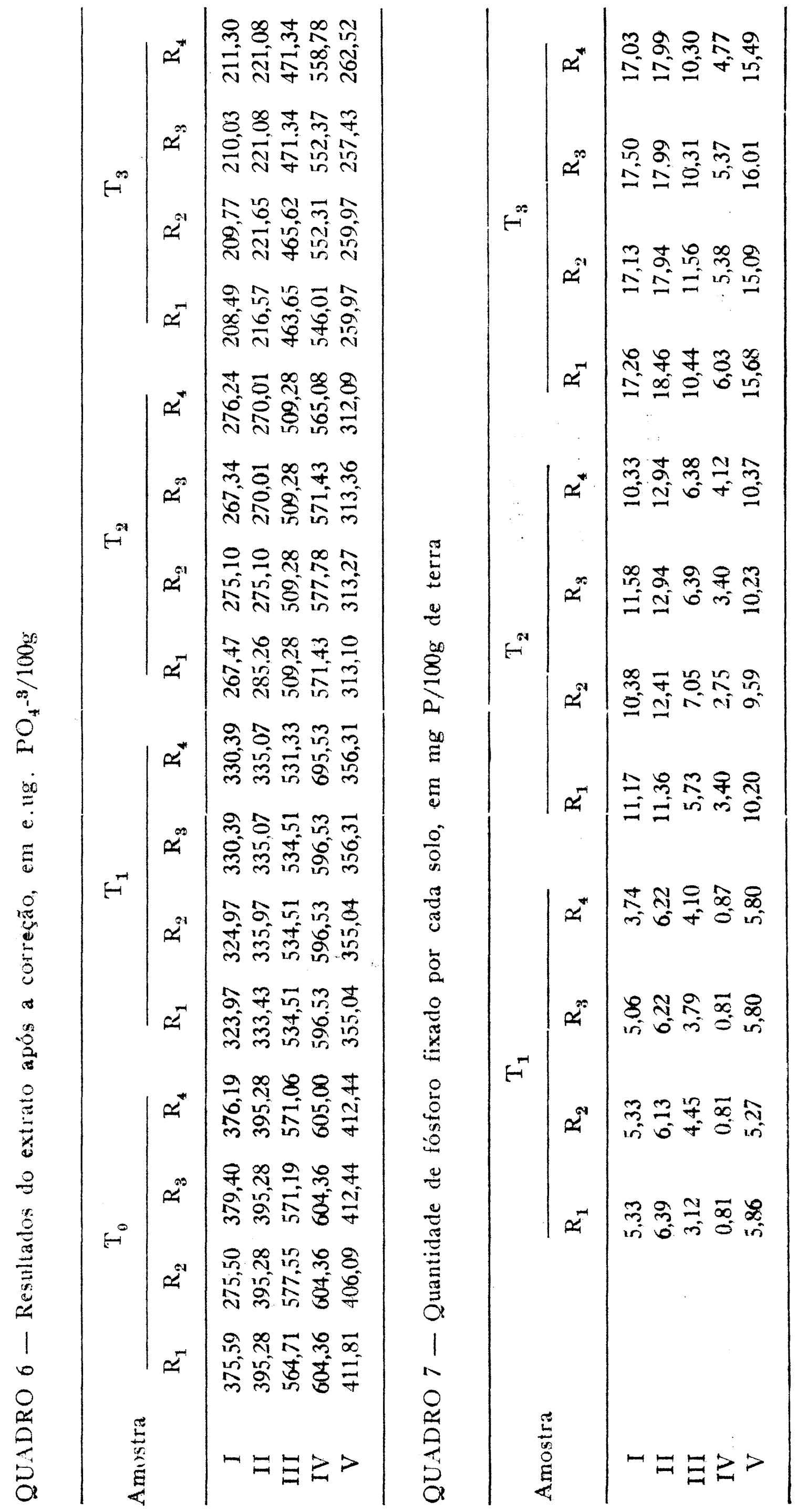




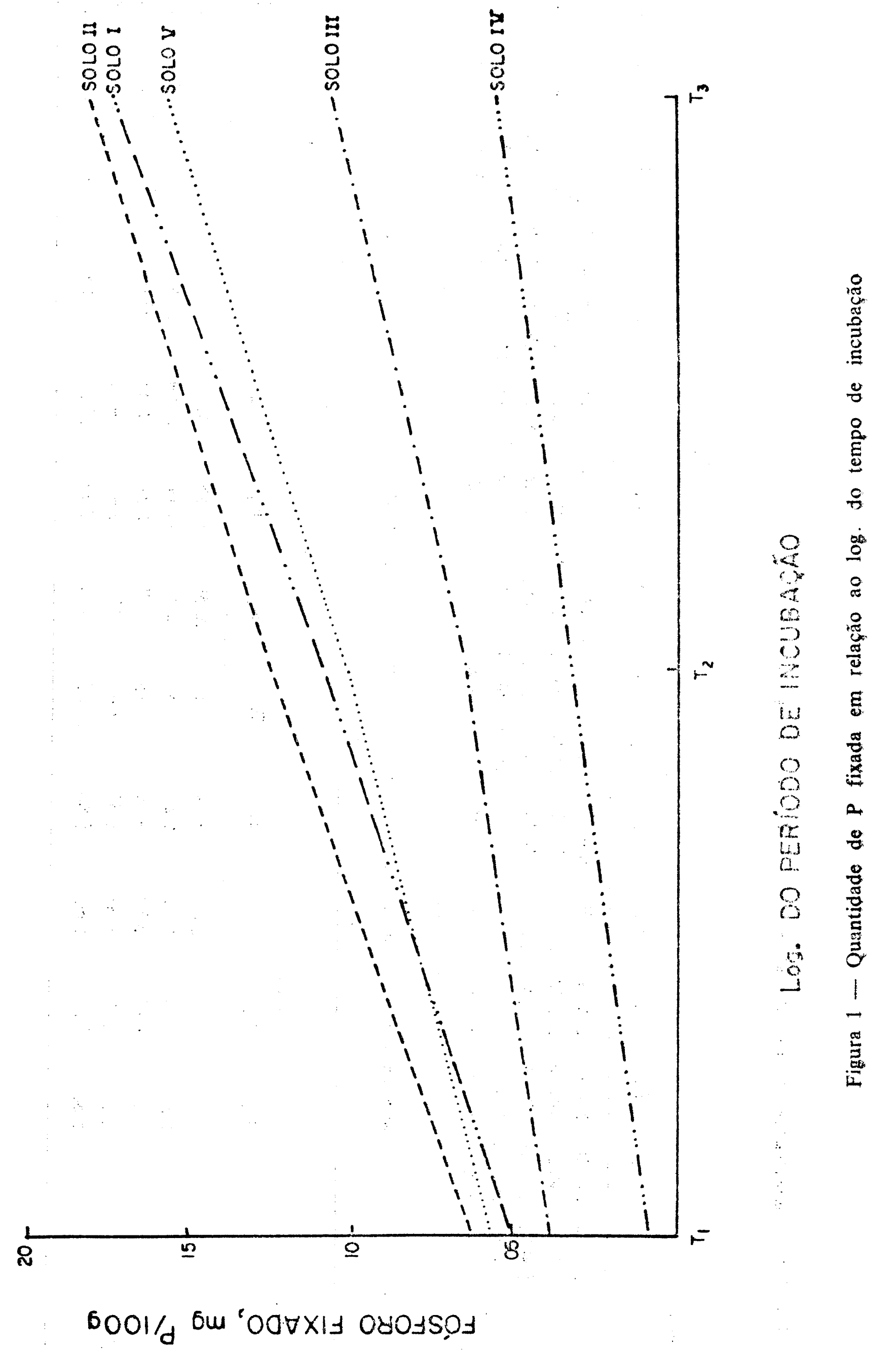


QUADRO 8 - Análise da variância

\begin{tabular}{|c|c|c|c|}
\hline Fonte de variação & GL & $Q M$ & $\mathrm{~F}^{2}$ \\
\hline \multicolumn{4}{|l|}{ - Solo I } \\
\hline Total & 11 & & \\
\hline Tratamentos & 2 & 146,96 & $918,50^{* *}$ \\
\hline Componente linear & (1) & $(67,59)$ & $(422,43)^{* *}$ \\
\hline Componente quadrática & (1) & $(226,33)$ & $(1.414,56)^{* *}$ \\
\hline Residuo & 9 & 0,16 & \\
\hline \multicolumn{4}{|l|}{ _- Solo II } \\
\hline Total & 11 & & \\
\hline Tratamentos & 2 & 140,60 & $703,10^{* *}$ \\
\hline Componente linear & (1) & $(64,37)$ & $(321,85)^{* *}$ \\
\hline Componente quadrática & (1) & $(216,87)$ & $(1.084,35)^{* *}$ \\
\hline Resíduo & 9 & 0,20 & \\
\hline \multicolumn{4}{|l|}{ - Solo III } \\
\hline Total & 11 & & \\
\hline Tratamentos & 2 & 47,08 & $147,10^{* *}$ \\
\hline Componente linear & (1) & $(39,94)$ & $(124,81)^{* *}$ \\
\hline Componente quadrática & (1) & $(54,22)$ & $(169,43)^{* *}$ \\
\hline Resíduo & 9 & 0,32 & \\
\hline \multicolumn{4}{|l|}{ - Solo IV } \\
\hline Total & 11 & & \\
\hline Tratamentos & 2 & 20,15 & $110,26^{* *}$ \\
\hline Componente linear & (1) & $(33,43)$ & $(175,94) * *$ \\
\hline Componente quadrática & (1) & $(8,47)$ & $(44,57)^{* *}$ \\
\hline Resíduo & 9 & 0,19 & \\
\hline \multicolumn{4}{|l|}{ - Solo V } \\
\hline Total & 11 & & \\
\hline Tratamentos & 2 & 98,08 & $891,63 * *$ \\
\hline Componente linear & (1) & $(55,97)$ & $(508,81)^{* *}$ \\
\hline Componente quadrática & (1) & $(140,20)$ & $(1.274,54)^{* *}$ \\
\hline Resíduo & 9 & 0,11 & \\
\hline
\end{tabular}

a* - significativo ao nível de $1 \%$. 
QUADRO 9 - Quantidade de $\mathrm{P}$ fixada por cada solo, médias das repetições nos 3 períodos estudados

\begin{tabular}{rc}
\hline Solo & P fixado, $\mathrm{mg} / 100 \mathrm{~g}$ de terra \\
\hline II & 12,24 \\
I & 11,06 \\
V & 10,45 \\
III & 6,96 \\
IV & 3,21 \\
\hline
\end{tabular}

d.m.s., Tukey a $5 \%=3,00$

\section{LITERATURA CITADA}

ANDRADE, S.S. de 1971. Gênese e dissertação de solos de três catenas nos municípios de Piracicaba e Rio Claro. Tese Mestrado. Piracicaba, E.S.A. "Luiz de Queiroz" 74 p. (Mimeografada).

BASS, G.B. \& H.D. SHIELING, 1950. Method for determining relative phosphate - fixing capacity of acid soils. Soil Sci. 69:269-280.

CAVALCANTI, F.J. de A., 1974. Alguns aspectos da fixação do fósforo por solos da região de Piracicaba. Dissertação Mestrado, Piracicaba, E.S.A. "Luiz de Queiroz" - USP. 62 p. (Mimeografada).

ENGELSTAD, O.P. \& S.E. ALLEN, 1971. Ammonium pyrophosphate and ammonium orthophosphate as phosphorus sources. Soil Sci. Soc. Amer. Proc. $35(6): 1002-1004$.

GOEDERT, W.J., J.F. PATELLA \& J.F.V. MORAES, 1971. Formas de fósforo num planossolo do Rio Grande do Sul e sua disponibilidade para a cultura do arroz irrigado. Pesq. agropec. bras., Sér. Agron. 6:39-43.

MALAVOLTA, E., O.J. CROCOMO, R.G. de ANDRADE, C. ALVIZURI, R. VENCOWSKY \& L.M.M. de FREITAS, 1965. Estudos sobre a fertilidade dos solos do cerrado; I Efeito da calagem na disponibilidade do fósforo (Nota prévia) Anais da E.S.A. "Luiz de Queiroz", 22:131-138.

MANDAL, L.N. \& R.K. DAS, 1970. Transformation of applied water solubre phosphate in acid lowland rice soils. Soil Sci. 110(1):263-267.

McLEAN, E.O. \& T.J. LOGAN, 1970. Phosphorus for plants grown in soils with differing phosphorus fixation tendencies: Soil Sci. Soc. Amer. Proc. 34:907-911.

RANZANI, G., O. FREIRE \& T. KINJO, 1966. Carta de Solos do Município de Piracicaba. Piracicaba, Centro de Estudos de Solos da E.S.A. "Luiz de Queiroz", USP, $85 \mathrm{p}$.

VETTYRI, L., 1966. Métodos de análise de solos. Divisão de Pedologia e Fertilidade do Solo. Rio de Janeiro, 19 p. (Mimeografaido).

WAUGH, D.L. \& J.W. FITTS, 1966. Estudos para interpretação de análises de solo; de laboratório e de vasos. Boletim Técnico n. 3 . International Soil Testing. 33 p. 


\section{SUMMARY}

\section{FACTORS THAT INTERFERE WITH PHOSPHORUS FIXATION I - INCUBATION PERIOD}

Research material for this work was soil samples from the plow layer from five soils. Samples were taken and submitted to different incubations periods $\left(T_{0}=0 ; T_{1}=2\right.$; $\mathrm{T}_{2}=20$ and $\mathrm{T}_{3}=200$ hours) after treatment with a monocalcium phosphate solution (4 $\mathrm{ml}$ of a solution with $1,500 \mathrm{ppm} P$ per 10 grams of soil).

Of the soils used nos. I, II and V were Latosols; whereas the others were Podzolics (Soils III and IV). At the end of each incubation period, the phosphorus content was determined using the extraction solution of $0.025 \mathrm{~N} \mathrm{H}_{2} \mathrm{SO}_{4}$ and $0.05 \mathrm{~N} \mathrm{HCl}$.

The quantity of fixed phosphorus was calculated by subtracting the values found for $T_{1}, T_{2}$ and $T_{3}$ from the "corrected strata" for $T_{0}$. For this purpose each soil sample was treated with an equal amount of destilled water and was observed at the four time periods specified above.

In accordance with the statistical analysis (Tukey's test) it was found that for each soil had a statistical significance at the level of $1 \%$ as related to the linear and quadratic effects of the incubation period. A joint analysis of the 5 soils samples Tukey's test at the 5\% level showed the lack of marked differences among Soils I, II and V (Latosols), but Soils of III and IV nos. (Podzolics) showed a statistical difference. 
